\title{
Cough and Cold Preparation
}

National Cancer Institute

\section{Source}

National Cancer Institute. Cough and Cold Preparation. NCI Thesaurus. Code C29630.

Synthetic compounds or mixtures that usually contain a combination of any of the following agents depending on the symptoms that need to be treated: an antitussive, a decongestant, an antihistamine, an analgesic antipyretic, an expectorant and/or an anticholinergic agent. A cough and cold preparation is usually administered orally to relieve symptoms of the cold, flu or allergy, such as cough, congestion, itchy, watery eyes, runny nose, sore throat and fever. 